\title{
Laser Evoked Bio-stimulation for Bone Fracture Healing (Case Report Study)
}

\author{
Emad Hamdy Guber*, Osama Fekry Ahmed** \\ * Consultant of Orthopedic surgery UN \& MOH Egypt \\ **National Institute of Laser Enhanced Science, Cairo University
}

\begin{abstract}
Summary: BACKGROUND: The stimulating effect of low level laser phototherapy on bone healing has been shown in a number of in vitro and animal studies. However, the effect of LLLT on the bone healing in human has not been previously wide demonstrated. The article reports an accidentally injury pattern and reported as fractures of the mid third of the left tibia.

OBJECTIVE: The purpose of this case study was to demonstrate the biological effects of low-level laser therapy (LLLT) on tibial fractures treated surgically using radiographic, examinations.

CASE REPORT: The case hospitalized for conventional surgery and followed by applying Low Level Laser Therapy LLLT to enhance and accelerate the bone fracture healing of the left tibia using the effect of laser bio-stimulation. Radiological x-ray imaging evaluation follow up for our case showed a significant bone healing rate as result of exposure of Diode Laser $650 \mathrm{~nm}$ with energy density of $5 \mathrm{~J} / \mathrm{cm}^{2}$ three times/week for one month. Radiographic findings revealed no significant fracture callus thickness difference before using LLLT however, the fractures showed significant callus formation after using LLLT.

CONCLUSION: The study suggests that LLLT accelerates the process of fracture repair or cause increases in callus volume
\end{abstract}

Keywords: Low Level Laser, Laser bio-stimulation, and bone fractures healing

Submitted date 31 May 2013

Accepted Date: 06 June 2013

\section{Introduction}

Low Level Laser Therapy (LLLT) is quickly becoming the first line of attack in pain control and tissue healing in rehabilitation medicine. It is safe, painless, quick and easy to apply and results are often immediate with no side effects. [1]

Low-level laser therapy (LLLT) is a biophysical form of intervention in the fracture-repair process, which, through several mechanisms, accelerates the healing of fractures and enhances callus formation. The effect of laser on fracture healing is controversial. Some authors affirm that LLLT can accelerate bone formation by increasing osteoblastic activity. The objective of our study was to evaluate the effect of laser therapy on fracture healing. [2]

Low-level laser therapy is a clinically well established tool for enhancement of wound healing. In vitro studies have also shown that low level laser therapy has a biostimulatory effect on cells of different origin [3]

Bone healing differs from that of soft tissues because of its morphology and composition Usually the healing process of bone is slower than that of soft tissues [4].

Several previous reports have shown that the use of laser photobiomodulation improves the trealment of bone defects. [5]

It is possible that LLLT effect on bone regeneration depends not only on the total dose of irradiation, but also on the irradiation time and the irradiation mode (Continuous or Pulsed). [6]

Low-level laser therapy is the application of red and near infrared light over injuries or wounds to improve soft tissue healing and relieve both acute and chronic pain. Low-level therap y u s e s cold (sub thermal) laser light energy to direct bio-stimulative light energy to the body's cells without injuring or damaging them in any way.

The therapy is precise and accurate; and offers safe and effective treatment for a wide variety of conditions. The energy range of low level laser light lies between 1 and $500 \mathrm{~mW}$ (milli watts), while for surgical lasers the energy range lies between 3000 and 10000 mWLow-level lasers, applied in the red spectral region and near infrared regions, promote cellular photobiomodulation effects and therapeutic responses induced by photochemical, photoelectric and photoenergetic reactions. 
The most commercially available lasers are the Helium-Neon (He-Ne), emitting wavelengths of $632.8 \mathrm{~nm}$, and the semiconductor diodes, such as gallium- arsenide (GaAs) and gallium-aluminum- arsenide

(GaAlAs), emitting wavelength of 650 and $830 \mathrm{~nm}$, respectively. Their characteristics include the absence of thermal and deleterious effects to the human body, promoting bio-stimulation of cells and thereby improving regenerative capacity. [1-5]

This paper reports recent follow up for tibia bone fracture case with delayed fracture healing, reporting the observations on the effect of LLLT on bone fracture healing.

\section{Clinical Case}

This was a 45 years-old male with left poliomyelitis victim of accidentally falling at home presenting injury at the mid third of the left tibia. At clinical examination, distal pulses were present, and no peripheral neurological deficit was found. X-ray at anteroposterior and lateral plane of the left tibia showed longitudinal oblique fractures at middle third of the tibia, associated with fracture of the neck of the left fibula (fig1).

The patient has been hospitalized for internal fixation of the middle third fracture of left tibia by Dynamic Compression Plate DCP (10 holes and 9 screws)

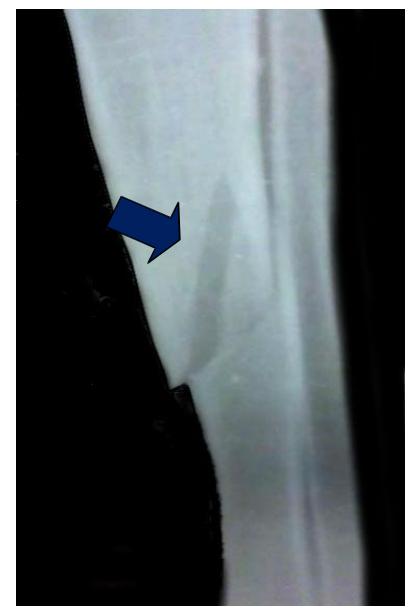

Fig (1) showed longitudinal oblique fracture at the mid third of the tibia.

\section{Results \& Discussion:}

Normally, the callus formation starts fixation then we canlnote it radiologically at the second week post surgery. The expected healing time for this case is from $6-8$ weeks. The controlled x-ray at 8 weeks showed just some callus formation with delayed healing fig (2)

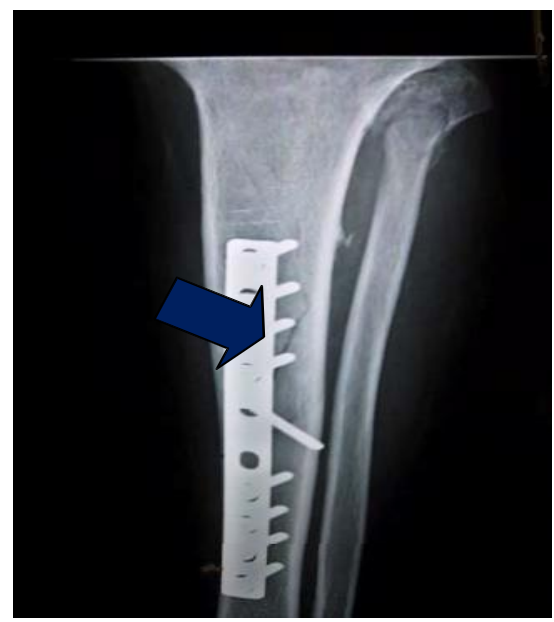

Fig (2) showed delayed bone healing \& callus formation. 
So we have continued follow up the case by using Low Leve Laser Therapy LLLT application for $\quad$ bone healing bio- stimulation. LLLT sessions were used applying energy density $5 \mathrm{~J} / \mathrm{cm}^{2}$ (6-7).

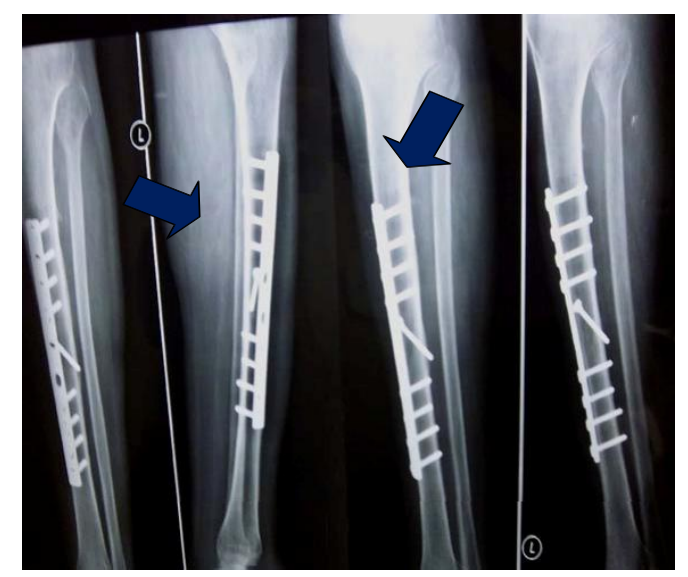

Fig (3) showed x-ray of complete healing at 12 week post surgery (4 weeks after LLLT application)

The controlled $\mathrm{x}$-ray at 12 week post surgery (4 weeks after LLLT application), showed good callus formation with great healing evidence fig (3).

In agreement with the present work Kazem et.al, 2010 demonstrated that the use of laser could enhance callus development in the early stage of the healing process, with doubtful improvement in biomechanical properties of the healing bone; therefore, laser therapy may be recommended as an additional treatment in non-union fractures in humans. [2]

In accordance with Pereira CL, et.al., 2009 who stated that Low-intensity laser therapy did not affect the area of bone formed within the threads, but it may improve bone-to-implant contact BIC in rabbit tibiae. This implies the important of improving BIC especially in some fractures that need good BIC. [8]

AboElsaad NS, et.al., 2009 have confirmed the positive effect of soft laser (LLLT) in accelerating bone regeneration, which have been showed in our study. [9]

\section{Conclusion}

The results of this case study suggest that LLT significantly accelerates bone regeneration and healing in humans. The irradiation parameters and protocol used in this study were successful in decreasing treatment time. As a consequence, fiture perspectives of this work are decreased treatment costs and less discomfort for the patient. Further studies are still required to explain the mechanisms of LILT and more clinical trials are necessary to optimize treatment parameters.

[1] Reddy GK. Photobiological basis and clinical role of low-intensity lasers in biology and medicine. Journal of Clinical Laser Medicine \& Surgery. 2004;22(2):141-50.

[2] Lasers Med Sci. 2010 Jan; 25(1):73- 7. Epub 2009 Apr 28 Effect of low-level laser therapy on the fracture healing process. Kazem Shakouri S, Soleimanpour J, Salekzamani Y, Oskuie MR. Tabriz University ofMedical Sciences,Tabriz, Iran. sk0531ir@yahoo.com

[3] In itial effects of low-level laser therapy on growth and differentiation of human steoblast-likecells. Stein E, Koehn J, Sutter W, Wendtlandt G, Wanschitz F, Thurnher D, Baghestanian M, Turhani D.Department of CranioMaxillofacial and Oral Surgery, Medical University of Vienna, Austria. Wien Klin Wochenschr. 2008; 120(3-4):112-7.

[4] Luger EJ, Rochkind S, Wollman Y, Kogan G, Dekel S. Effect of low-power laser irradiation on the mechanical properties of bone fracture healing in rats. Lasers Surg Med 1998; 22:97-102.

[5] Infrared laser light further improves bone healing when associated with bone morphogenic proteins: an in vivo study in a rodent model. Gerbi ME, Marques AM, Ramalho LM, Ponzi EA, Carvalho CM, Santos Rde C, Oliveira PC, Nóia M, Pinheiro AL. School of Dentistry, Federal University of Pernambuco, Recife, PE, Salvador. Photomed Laser Surg. 2008 Feb;26(1):55-60.

[6]. Sommer AP, Pinheiro ALB, Mester A, Franke RP, and Whelan, HT (2001): Biostimulatory windows in low intensity laser activation: Lasers, Scanners and NASA's Light Emitting Diode Array System. Journal Clinical Laser Medicine and Surgery, 19:29- 34 .

[7] Kawasaki K, Shimizu N. Effects of low-energy laser irradiation on bone remodeling during experimental tooth movementinrats.Laser SurgMed 2000;26(3):282-91.

[8] Pereira CL, Sallum EA, Nociti FH Jr, Moreira RW. The effect of low-intensity laser therapy on bone healing around titanium implants: a histometric study in rabbits. Int J Oral Maxillofac Implants. 2009 Jan-Feb;24(1):47-51.

[9] AboElsaad NS, Soory M, Gadalla LM, Ragab LI, Dunne S, Zalata KR, Louca C. Effect of soft laser and bioactive glass on bone regeneration in the treatment of bone defects (an experimental study). Lasers Med Sci. 2009 Jul;24(4):527-33. E pub 2008 Jul 15. 